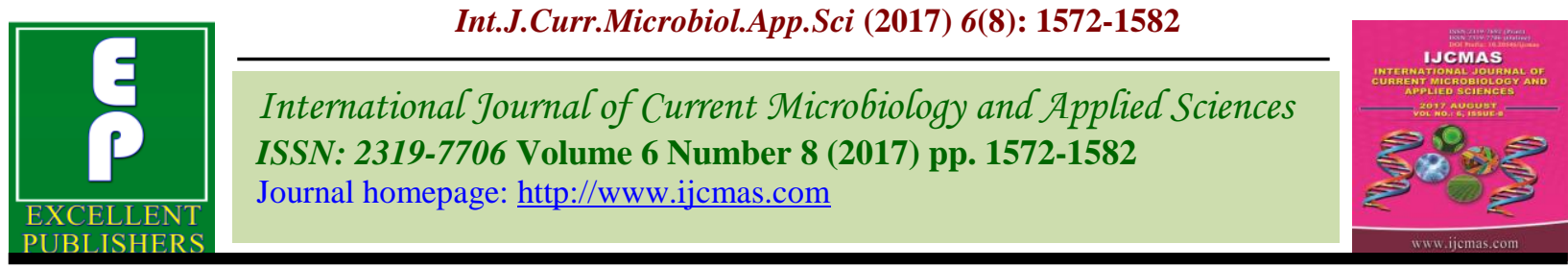

Original Research Article

https://doi.org/10.20546/ijcmas.2017.608.188

\title{
Effect of Organic Resources of Nutrients for Corms and Cormlet Production of Three Cultivars of Gladiolus (Gladiolus grandiflorus L.) in the Hilly Tract of West Bengal, India
}

\author{
I. Sarkar*, S. Maitra and K. Swathi \\ Department of Floriculture and Landscaping, Uttar Banga Krishi \\ ViswavidyalayaPundibari, Coochbehar, India \\ *Corresponding author
}

\section{A B S T R A C T}

The experiment was carried out in two consecutive years 2009 and 2010 at Regional Research Station (Hill zone) of Uttar Banga Krishi Viswavidyalaya, Kalimpong, Darjeeling, West Bengal to study the effect of organic resources of nutrients for corms and cormlet production of three cultivars of gladiolus (Gladiolus sp.) namely Candyman, American Beauty and Jester. The different organic sources of nutrients namely bone meal

Keywords

Gladiolus, Organic, Corm and Cormlet.

Article Info

Accepted:

17 June 2017

Available Online:

10 August 2017 (5t/ha), Farm yard manure (FYM) (50t/ha), vermicompost $(5 \mathrm{t} / \mathrm{ha})$ were used alone and in combination with bio-fertilizers namely [VAM] (2g/plant), Phosphobacteria (2g/plant) and Azospirillum (2g/plant). The cv. Jester performed better in production of number of corm per plant (2.28), equatorial diameter of corm $(6.54 \mathrm{~cm})$, average weight of corm $(62.94 \mathrm{~g})$ and average weight of cormlet $(38.41 \mathrm{~g})$. The higher polar diameter of corm $(3.79 \mathrm{~cm})$ and greater number of cormlet per plant (125.01) were produced by the cv. Candyman. Among the different treatments of manures and bio-fertilizers the application of FYM $(25 \mathrm{t} / \mathrm{ha})$, vermicompost (2.5t/ha), bone meal (5t/ha) and Trichoderma @20g/m ${ }^{2}\left(\mathrm{~T}_{4}\right)$ produced greater number of corm per plant (3.07), equatorial diameter of corms $(6.42 \mathrm{~cm})$ and number of cormlet per plant (123.83). On the other hand, the treatment consisting of bone meal @ 5t/ha, VAM, Phosphobacteria and Azospirillum each @ 2g/plants and Trichoderma@20g/ $\mathrm{m}^{2}$ in $\mathrm{T}_{9}$ was found better in respect of polar diameter of corm (3.90 $\mathrm{cm})$ and average weight of corm $(65.0 \mathrm{~g})$. Among the combination of treatments, the propagation co-efficient was found maximum with the cv. Jester $(495.60 \%)$ in the case of T9 (VAM @ 2g/plant + Phosphobacteria @ 2g/plant + Azospirillum @ 2g/plant + Bone meal @5t/ha + Trichoderma@20g/ $\mathrm{m}^{2}$ ).

\section{Introduction}

Floriculture has become a profitable industry and foreign exchange earner. Gladiolus is an important floriculture crop in India for its breathtaking beauty with widest range of magnificent colour, size and form of flowers. Botanically the modern hybrids are known as Gladiolus grandiflorus Linn. It belongs to the family Iridaceae. In West Bengal, Darjeeling district ranked first in gladiolus production from a quite long time and it has also a good tradition for gladiolus cultivation. Gladiolus being a bulbous plant require a fairly good amount of nutrients (Both macro and micro element). Recent trends on eco-friendly, sustainable commercial practices improved Integrated Nutrient Management (INM) as a 
major component considering the excessive use of chemical fertilizer leading to the depletion of soil inherent fertility and also loss of structural and textural configuration. Residual availability of chemical fertilizer in the cut flower often possess problem during export because the developed world already inclines towards the products developed from organic cultivation. Besides organic cultivation restore the soil fertility improved soil health, provides nutrients judiciously throughout the crop cultivation period and also cost compatibility. In addition it improves the quality of the flowers, depth and brightness of colour, rigidity of the stem and obviously the post-harvest keeping quality of flower. Bio-fertilizers are important component of organic cultivation. The inherent tendency of using a small amount of organic manure by the gladiolus farming community of this region will be utilized to implement the proper organic cultivation of gladiolus through development of the appropriate organic cultivation protocol of gladiolus for this region which is still not pinpointed. The major component of organic farming like (VAM), Bacterial fertilizer, vermicompost, FYM etc. will be used both singly and in combination to develop the proper organic cultivation schedule of some promising varieties of this region. (VAM), Trichoderma, Azospirillum, FYM, Phosphobacteria, Vermi-Compost, Bone Meal was tested either singly or in combination to develop the organic cultivation practices of Gladiolus in this region. In view of the above, research work has been carried out with title "Effect of organic resources of nutrients for corms and cormlet production of three cultivars of gladiolus (Gladiolus grandiflorus L.) in the hilly tract of West Bengal."

\section{Materials and Methods}

The present investigation was carried out during 2009 and 2010 in the Regional
Research Station (Hill Zone), Uttar Banga Krishi Viswavidyalaya, Kalimpong, Darjeeling, West Bengal. The farm is situated in the Northern part of the state lying between $20^{\circ} 31^{\prime}$ and $27^{0} 31^{\prime}$ North latitudes and between $87^{0} 59^{\prime}$ and $88^{0} 53^{\prime}$ East longitude at an altitude of 1250 metres above mean sea level., of which $80 \%$ was received during June to September. The average maximum and minimum temperature round the year recorded $27^{\circ}$ and $3^{\circ} \mathrm{C}$ respectively and the average annual rainfall varies from 2100 to $2700 \mathrm{~mm}$. The relative humidity also varies from 70-95\% depending upon the locality and season of the year. The soil is mostly categorized as brown forest soil due to their characteristic reddish brownish colour. The soil reaction varies from highly acidic to neutral in nature. The soil reaction of the study area was analyzed as highly acidic $(\mathrm{pH}$ 5.98). The No. of treatment combinations was 30 and the plot size was $1 \mathrm{~m} \mathrm{X} 1 \mathrm{~m}$ (A space of $50 \mathrm{~cm}$. in both ways between 2 plots is left). Planting of corms were done with the spacing of $25 \mathrm{~cm} \times 20 \mathrm{~cm}$ (20 plants per plot) by following the Statistical Design of Factorial RBD with 2 factors replicated thrice. Three cultivars of gladiolus (Gladiolus sp.) namely Candyman, American Beauty and Jester were taken as first factor and ten treatments were taken as (i) without fertilizer and organic supplements, (ii) Bone meal (5t/ha) + Trichoderma $\left(20 \mathrm{~g} / \mathrm{m}^{2}\right)$ as basal, (iii) FYM $(50 \mathrm{t} / \mathrm{ha})+$ bone meal $(5 \mathrm{t} / \mathrm{ha})+$ Trichoderma $\left(20 \mathrm{~g} / \mathrm{m}^{2}\right)$ as basal, (iv) Vermicompost (5t/ha) + bone meal $(5 \mathrm{t} / \mathrm{ha})+$ Trichoderma $\left(20 \mathrm{~g} / \mathrm{m}^{2}\right)$ as basal, (v) 1/2 FYM + 1/2 Vermicompost + bone meal $(5 \mathrm{t} / \mathrm{ha})+$ Trichoderma $\left(20 \mathrm{~g} / \mathrm{m}^{2}\right)$ as basal, (vi) VAM (2g/plant) + bone meal $(5 \mathrm{t} / \mathrm{ha})+$ Trichoderma $\left(20 \mathrm{~g} / \mathrm{m}^{2}\right)$ as basal, (vii) Azospirillum (2g/plant) + bone meal (5t/ha) + Trichoderma $\left(20 \mathrm{~g} / \mathrm{m}^{2}\right)$ as basal, (viii) Phosphobacteria (2g/plant) + bone meal $(5 \mathrm{t} / \mathrm{ha})+$ Trichoderma $\left(20 \mathrm{~g} / \mathrm{m}^{2}\right)$ as basal, (ix) VAM (2g/plant) + Azospirillum (2g/plant) + bone meal $(5 \mathrm{t} / \mathrm{ha})+$ Trichoderma $\left(20 \mathrm{~g} / \mathrm{m}^{2}\right)$ as 
basal, (x) VAM (2g/plant) + Azospirillum $(2 \mathrm{~g} /$ plant $)+$ Phosphobacteria (2g/plant) + bone meal $(5 \mathrm{t} / \mathrm{ha})+$ Trichoderma $\left(20 \mathrm{~g} / \mathrm{m}^{2}\right)$ as basal as second factor.

The observations on different parameters were recorded from time to time in the experiment conducted. Best growing five plants in each replication were tagged for the purpose of recording observations, leaving the first and last plant in a row. The corm and cormlet related attributes of the experiment have been taken as and when necessary. The experimental data were analyzed statistically by the technique of analysis of variance (ANOVA) as applicable to RBD (Gomez and Gomez, 1984) using MSTAT - C software to find the significance levels of each of the parameters. Critical Difference (CD) values at $5 \%$ level of significance were calculated for the parameters in which "F-test" was found significant.

\section{Results and Discussion}

The observations recorded at the successive stage of the plant development were analyzed statistically and was presented in the tables. The experimental findings of the present investigation and discussion had been done with reference by different authors as corelated with the different parameters. The results of the different parameters exerted by different treatments are stated below. Jester was found to have the more number of corms per plant (2.17) and Candyman was found the least (1.71) in the year 2009. In the 2010 and pooled result also, Jester was found to have the more number of corms (2.39) and (2.28) respectively and Candyman (1.79) in 2010 and in the pooled result also, Candyman was found to have the lowest number of corms (1.75) per plant which was presented in the table 1 and figure 1. Candyman (124.37) produced maximum number of cormels in the year 2009, in 2010 (125.65) and in the pooled result (125.01). American Beauty produced maximum (87.82) number of cormels per plant in the year 2009, and in 2010 (89.24) and (88.53) in the pooled result respectively (Table 1 and Fig. 2).

In the year 2009, the highest weight of corm was found in the variety Jester $(62.63 \mathrm{~g})$ and the lowest weight of corm was found in American Beauty (49.25 g). In the year 2010 same result like, Jester was found the highest weight $(63.25 \mathrm{~g})$ and American Beauty had the lowest weight $(49.54 \mathrm{~g})$. In the pooled result also, Jester $(62.94 \mathrm{~g})$ was found to have the highest and American Beauty (49.40 g) was found to have the lowest weight of corm among the three varieties taken for the investigation (Table 1). In the year 2009, American Beauty produced the lowest cormels per plant (21.04 g) and Jester was found to have the highest cormels per plant $(37.72 \mathrm{~g})$. In the year 2010 and pooled result also, American Beauty was found to have the lowest weight of cormels per plant (22.16 g) and $(21.60 \mathrm{~g})$ respectively. In the year 2009, Jester was found to have the highest weight of cormels (37.72 g), in 2010 (39.10 g) and pooled result $(38.41 \mathrm{~g})$ also (Table 2$)$.

Table 2 revealed that Candyman showed the highest polar diameter of corm $(3.86 \mathrm{~cm})$ in 2009, $(3.73 \mathrm{~cm})$ in 2010 and in the pooled result $(3.79 \mathrm{~cm})$. The lowest polar diameter of corm was found in American Beauty in 2009 $(2.95 \mathrm{~cm})$, in $2010(3.07 \mathrm{~cm})$ and in the pooled result $(3.01 \mathrm{~cm})$ also. In the year 2010 , Jester $(3.26 \mathrm{~cm})$ was found to be statistically at par with American Beauty $(3.07 \mathrm{~cm})$. In the year 2009, Jester was found to have the highest equatorial diameter of corm $(6.37 \mathrm{~cm})$ and the lowest equatorial diameter of corm $(3.99 \mathrm{~cm})$ with the var. American Beauty. In the year 2010 and pooled result also, American Beauty was found to have the lowest diameter $(4.48 \mathrm{~cm})$ and $(4.23 \mathrm{~cm})$ respectively. Jester was found to have the 
highest diameter $(6.54 \mathrm{~cm})$ in the pooled result also (Table 2). Jester $(378.50 \%)$ was found to have the highest propagation coefficient in the year 2009. It was also found the highest in the year 2010 (395.30\%) and in the pooled result $(386.90 \%)$ also. Candyman $(223.79 \%)$ was found to be the lowest in the year 2009. It was also the lowest in the year $2010(235.03 \%)$ and in the pooled result
(229.41\%). $\mathrm{T}_{0}(159.91 \%)$ was found to have the lowest propagation co-efficient among all 10 treatments. It was also found to have the lowest result in the year $2010(165.44 \%)$ and in the pooled result $(162.67 \%)$. The highest propagation co-efficient was found in $\mathrm{T}_{9}$ $(432.87 \%)$ in $2009,2010(462.86 \%)$ and in the pooled result $(447.86 \%)$ in the table 3 and figure 3 .

Fig.1 Effect of organic resources of nutrients on no. of corms per plant (pooled)

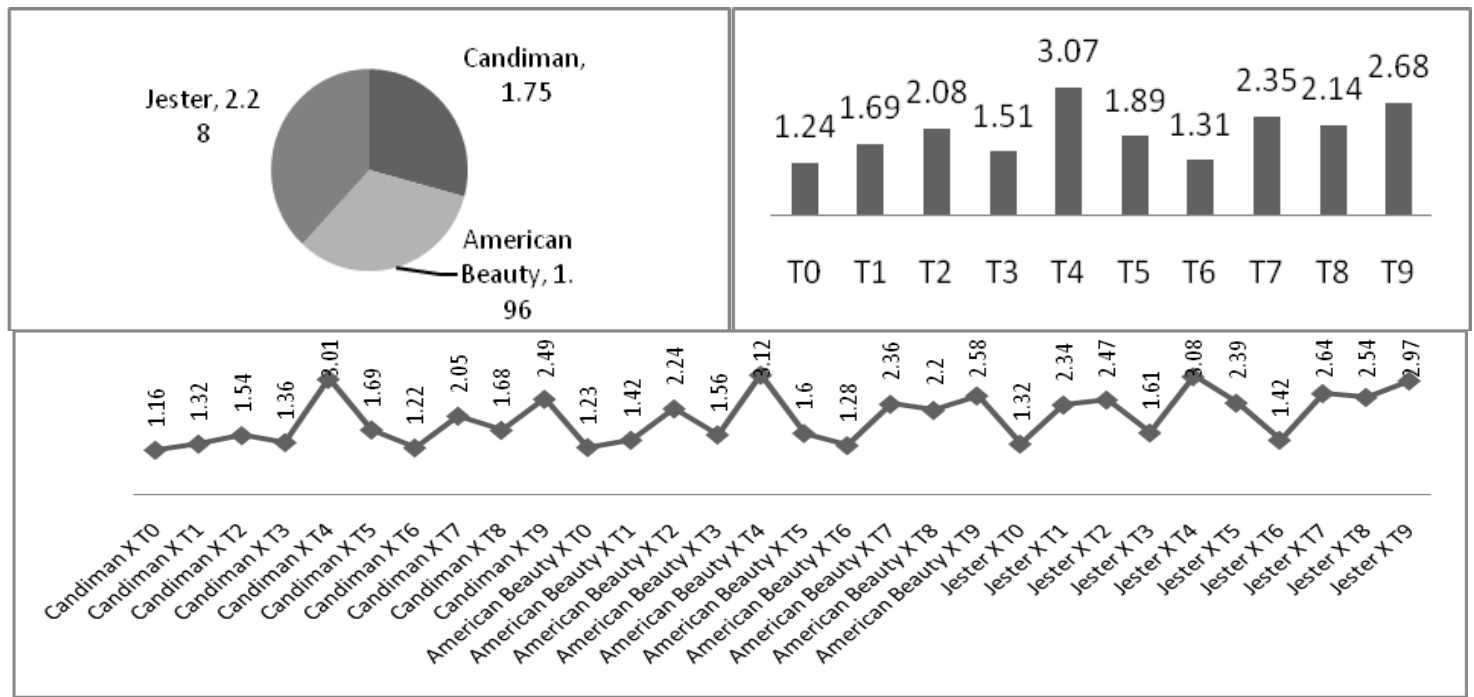

Fig.2 Effect of organic resources of nutrients on no. of cormels per plant (pooled)

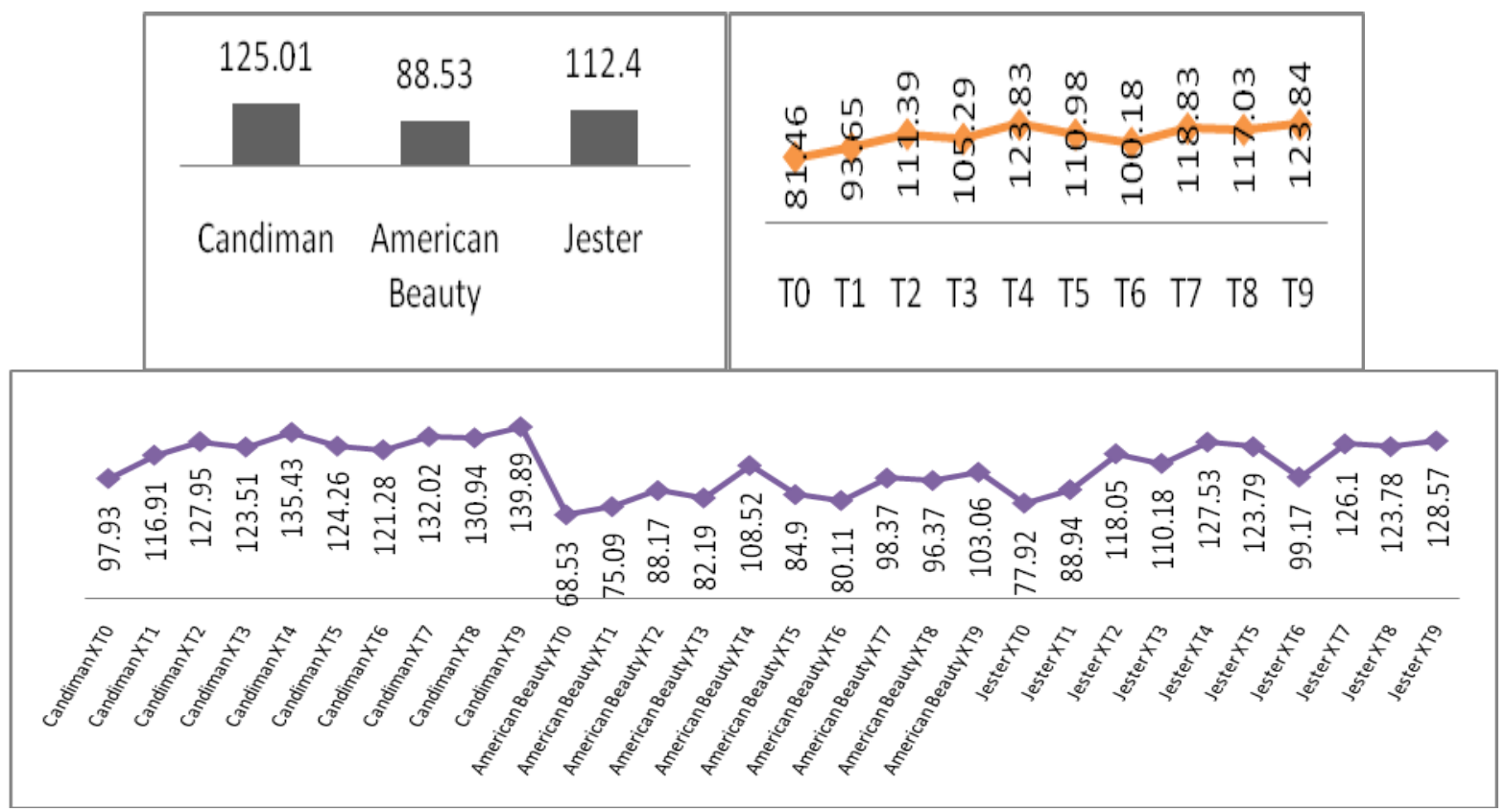


Fig.3 Effect of organic resources of nutrients on propagation co-efficient (\%) (pooled)

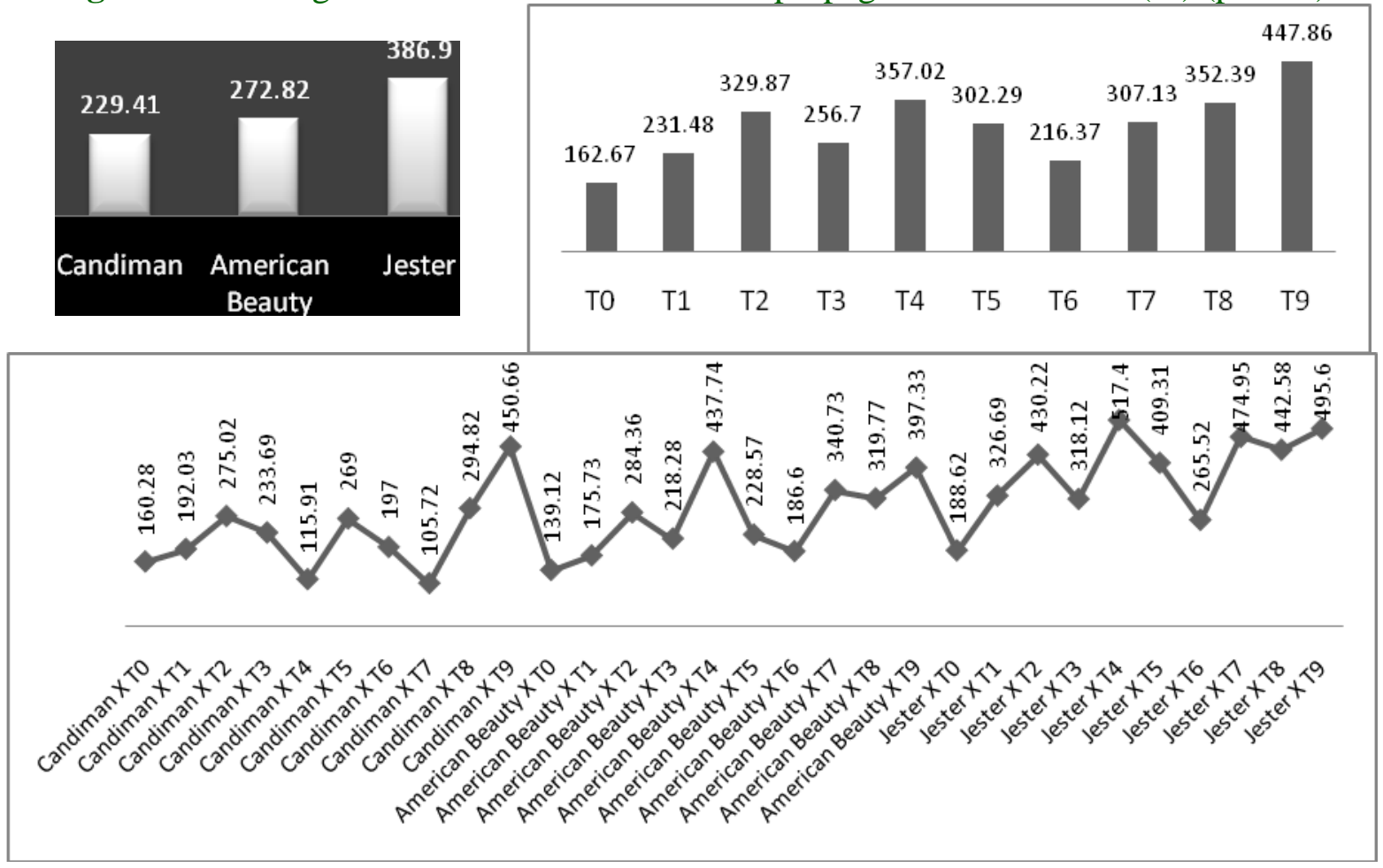

Table.1 Effect of organic resources of nutrients on no. of corms per plant, no. of cormels per plant and weight of the corm (g)

\begin{tabular}{|c|c|c|c|c|c|c|c|c|c|}
\hline \multirow[t]{2}{*}{ Treatment Details } & \multicolumn{3}{|c|}{ NO. OF CORMS PER PLANT } & \multicolumn{3}{|c|}{$\begin{array}{c}\text { NO. OF CORMELS PER } \\
\text { PLANT }\end{array}$} & \multicolumn{3}{|c|}{$\begin{array}{l}\text { WEIGHT OF THE } \\
\operatorname{CORM(g)}\end{array}$} \\
\hline & 2009 & 2010 & Pooled & 2009 & 2010 & Pooled & 2009 & 2010 & Pooled \\
\hline \multicolumn{10}{|c|}{ Cultivar(C) } \\
\hline Candyman & 1.71 & 1.79 & 1.75 & 124.37 & 125.65 & 125.01 & 53.81 & 54.23 & 54.02 \\
\hline American Beauty & 1.89 & 2.03 & 1.96 & 87.82 & 89.24 & 88.53 & 49.25 & 49.54 & 49.40 \\
\hline Jester & 2.17 & 2.39 & 2.28 & 110.96 & 113.85 & 112.40 & 62.63 & 63.25 & 62.94 \\
\hline S. Em \pm & 0.04 & 0.03 & 0.03 & 0.23 & 0.98 & 0.50 & 0.25 & 0.21 & 0.17 \\
\hline C.D. at $5 \%$ & 0.12 & 0.09 & 0.06 & 0.65 & 2.77 & 1.41 & 0.72 & 0.61 & 0.46 \\
\hline \multicolumn{10}{|c|}{ Treatments(T) } \\
\hline $\mathrm{T}_{0}$ & 1.11 & 1.36 & 1.24 & 80.82 & 82.10 & 81.46 & 40.52 & 40.87 & 40.69 \\
\hline $\mathrm{T}_{1}$ & 1.55 & 1.84 & 1.69 & 93.14 & 94.15 & 93.65 & 45.21 & 45.53 & 45.37 \\
\hline $\mathrm{T}_{2}$ & 1.97 & 2.19 & 2.08 & 110.88 & 111.90 & 111.39 & 58.22 & 59.50 & 58.86 \\
\hline $\mathrm{T}_{3}$ & 1.53 & 1.49 & 1.51 & 104.74 & 105.85 & 105.29 & 52.82 & 54.58 & 53.70 \\
\hline $\mathrm{T}_{4}$ & 3.03 & 3.11 & 3.07 & 122.80 & 124.85 & 123.83 & 64.74 & 63.80 & 64.27 \\
\hline $\mathrm{T}_{5}$ & 1.78 & 2.01 & 1.89 & 107.45 & 114.52 & 110.98 & 54.07 & 55.04 & 54.55 \\
\hline $\mathrm{T}_{6}$ & 1.30 & 1.31 & 1.31 & 99.62 & 100.75 & 100.18 & 49.46 & 50.61 & 50.03 \\
\hline $\mathrm{T}_{7}$ & 2.26 & 2.43 & 2.35 & 118.11 & 119.55 & 118.83 & 62.83 & 62.20 & 62.52 \\
\hline
\end{tabular}


Int.J.Curr.Microbiol.App.Sci (2017) 6(8): 1572-1582

\begin{tabular}{|c|c|c|c|c|c|c|c|c|c|}
\hline $\mathrm{T}_{8}$ & 2.09 & 2.18 & 2.14 & 116.33 & 117.73 & 117.03 & 59.22 & 59.81 & 59.51 \\
\hline $\mathrm{T}_{9}$ & 2.61 & 2.75 & 2.68 & 123.28 & 124.40 & 123.84 & 65.20 & 64.81 & 65.00 \\
\hline S. Em \pm & 0.08 & 0.06 & 0.05 & 0.42 & 1.79 & 0.92 & 0.46 & 0.39 & 0.30 \\
\hline C.D. at $5 \%$ & 0.22 & 0.17 & 0.14 & 1.19 & 5.07 & 2.57 & 1.31 & 1.10 & 0.85 \\
\hline \multicolumn{10}{|c|}{$\mathbf{C X T}$} \\
\hline Candyman $\mathrm{X} \mathrm{T}_{0}$ & 1.03 & 1.28 & 1.16 & 97.52 & 98.33 & 97.93 & 40.61 & 41.45 & 41.03 \\
\hline Candyman $\mathrm{X} \mathrm{T}_{1}$ & 1.13 & 1.51 & 1.32 & 116.43 & 117.39 & 116.91 & 45.42 & 44.37 & 44.90 \\
\hline Candyman $\mathrm{X} \mathrm{T}_{2}$ & 1.50 & 1.57 & 1.54 & 127.41 & 128.48 & 127.95 & 56.44 & 57.37 & 56.91 \\
\hline Candyman $\mathrm{X} \mathrm{T}_{3}$ & 1.44 & 1.28 & 1.36 & 122.60 & 124.43 & 123.51 & 50.49 & 52.69 & 51.59 \\
\hline Candyman $\mathrm{X} \mathrm{T}_{4}$ & 2.99 & 3.03 & 3.01 & 134.12 & 136.73 & 135.43 & 62.30 & 61.59 & 61.95 \\
\hline Candyman $\mathrm{X} \mathrm{T}_{5}$ & 1.61 & 1.77 & 1.69 & 123.82 & 124.71 & 124.26 & 52.24 & 54.49 & 53.36 \\
\hline Candyman $\mathrm{X} \mathrm{T}_{6}$ & 1.23 & 1.22 & 1.22 & 120.70 & 121.85 & 121.28 & 45.63 & 46.69 & 46.16 \\
\hline Candyman $\mathrm{X} \mathrm{T}_{7}$ & 2.01 & 2.09 & 2.05 & 131.36 & 132.68 & 132.02 & 61.58 & 60.65 & 61.12 \\
\hline Candyman $\mathrm{X} \mathrm{T}_{8}$ & 1.67 & 1.69 & 1.68 & 130.29 & 131.58 & 130.94 & 57.69 & 59.46 & 58.57 \\
\hline Candyman $\mathrm{X} \mathrm{T}_{9}$ & 2.49 & 2.49 & 2.49 & 139.46 & 140.32 & 139.89 & 65.65 & 63.58 & 64.61 \\
\hline American Beauty $\mathrm{X} \mathrm{T}_{0}$ & 1.11 & 1.35 & 1.23 & 67.58 & 69.48 & 68.53 & 37.31 & 38.59 & 37.95 \\
\hline American Beauty $\mathrm{X} \mathrm{T}_{1}$ & 1.27 & 1.56 & 1.42 & 74.49 & 75.68 & 75.09 & 40.70 & 41.48 & 41.09 \\
\hline American Beauty $\mathrm{X} \mathrm{T}_{2}$ & 2.09 & 2.39 & 2.24 & 87.60 & 88.75 & 88.17 & 50.64 & 54.42 & 52.53 \\
\hline American Beauty $\mathrm{X} \mathrm{T}_{3}$ & 1.41 & 1.71 & 1.56 & 81.72 & 82.66 & 82.19 & 47.38 & 49.53 & 48.46 \\
\hline American Beauty $\mathrm{X} \mathrm{T}_{4}$ & 3.07 & 3.17 & 3.12 & 107.60 & 109.43 & 108.52 & 57.35 & 56.43 & 56.89 \\
\hline American Beauty $\mathrm{X} \mathrm{T}_{5}$ & 1.63 & 1.58 & 1.60 & 84.31 & 85.49 & 84.90 & 48.25 & 48.38 & 48.31 \\
\hline American Beauty $\mathrm{X} \mathrm{T}_{6}$ & 1.32 & 1.24 & 1.28 & 79.52 & 80.69 & 80.11 & 44.28 & 45.35 & 44.82 \\
\hline American Beauty $\mathrm{X} \mathrm{T}_{7}$ & 2.22 & 2.49 & 2.36 & 97.50 & 99.23 & 98.37 & 56.43 & 53.32 & 54.87 \\
\hline American Beauty $\mathrm{X} \mathrm{T}_{8}$ & 2.22 & 2.18 & 2.20 & 95.29 & 97.45 & 96.37 & 51.64 & 50.51 & 51.08 \\
\hline American Beauty $\mathrm{X} \mathrm{T}_{9}$ & 2.58 & 2.58 & 2.58 & 102.57 & 103.55 & 103.06 & 58.53 & 57.39 & 57.96 \\
\hline Jester $\mathrm{X} \mathrm{T} \mathrm{T}_{0}$ & 1.19 & 1.45 & 1.32 & 77.35 & 78.49 & 77.92 & 43.63 & 42.56 & 43.10 \\
\hline Jester $\mathrm{X} \mathrm{T}_{1}$ & 2.24 & 2.44 & 2.34 & 88.50 & 89.39 & 88.94 & 49.52 & 50.73 & 50.12 \\
\hline Jester $\mathrm{X} \mathrm{T}_{2}$ & 2.32 & 2.62 & 2.47 & 117.63 & 118.47 & 118.05 & 67.58 & 66.72 & 67.15 \\
\hline Jester $\mathrm{X} \mathrm{T}_{3}$ & 1.75 & 1.47 & 1.61 & 109.89 & 110.47 & 110.18 & 60.61 & 61.52 & 61.06 \\
\hline Jester $\mathrm{X} \mathrm{T}_{4}$ & 3.03 & 3.14 & 3.08 & 126.69 & 128.38 & 127.53 & 74.58 & 73.37 & 73.98 \\
\hline Jester $\mathrm{X} \mathrm{T}_{5}$ & 2.09 & 2.69 & 2.39 & 114.21 & 133.36 & 123.79 & 61.71 & 62.25 & 61.98 \\
\hline Jester $\mathrm{X} \mathrm{T}_{6}$ & 1.37 & 1.48 & 1.42 & 98.63 & 99.71 & 99.17 & 58.48 & 59.78 & 59.13 \\
\hline Jester $\mathrm{X} \mathrm{T}_{7}$ & 2.56 & 2.72 & 2.64 & 125.46 & 126.74 & 126.10 & 70.48 & 72.63 & 71.56 \\
\hline Jester $\mathrm{X} \mathrm{T}_{8}$ & 2.39 & 2.69 & 2.54 & 123.40 & 124.16 & 123.78 & 68.31 & 69.47 & 68.89 \\
\hline Jester $\mathrm{X} \mathrm{T}_{9}$ & 2.75 & 3.19 & 2.97 & 127.80 & 129.34 & 128.57 & 71.41 & 73.45 & 72.43 \\
\hline S. Em \pm & 0.13 & 0.11 & 0.08 & 0.73 & 3.10 & 1.59 & 0.80 & 0.68 & 0.52 \\
\hline C.D. at $5 \%$ & 0.37 & 0.30 & 0.24 & 2.06 & 8.77 & 4.46 & 2.27 & 1.91 & 1.47 \\
\hline
\end{tabular}


Table.2 Effect of organic resources of nutrients on weight of cormels per plant (g), polar diameter of corm $(\mathrm{cm})$ and equatorial diameter of corm $(\mathrm{cm})$

\begin{tabular}{|c|c|c|c|c|c|c|c|c|c|}
\hline \multirow[t]{2}{*}{ Treatment Details } & \multicolumn{3}{|c|}{$\begin{array}{c}\text { WEIGHT OF CORMELS PER } \\
\text { PLANT(g) }\end{array}$} & \multicolumn{3}{|c|}{$\begin{array}{c}\text { POLAR DIAMETER OF } \\
\text { CORM }(\mathrm{cm})\end{array}$} & \multicolumn{3}{|c|}{$\begin{array}{l}\text { EQUATORIAL } \\
\text { DIAMETER OF } \\
\text { CORM }(\mathrm{cm})\end{array}$} \\
\hline & 2009 & 2010 & Pooled & 2009 & 2010 & Pooled & 2009 & 2010 & Pooled \\
\hline \multicolumn{10}{|c|}{ Cultivar(C) } \\
\hline Candyman & 35.98 & 37.16 & 36.57 & 3.86 & 3.73 & 3.79 & 5.69 & 5.59 & 5.64 \\
\hline American Beauty & 21.04 & 22.16 & 21.60 & 2.95 & 3.07 & 3.01 & 3.99 & 4.48 & 4.23 \\
\hline Jester & 37.72 & 39.10 & 38.41 & 3.23 & 3.26 & 3.25 & 6.37 & 6.72 & 6.54 \\
\hline S. Em \pm & 0.26 & 0.20 & 0.16 & 0.07 & 0.07 & 0.05 & 0.08 & 0.10 & 0.06 \\
\hline C.D. at $5 \%$ & 0.73 & 0.58 & 0.46 & 0.19 & 0.21 & 0.14 & 0.24 & 0.28 & 0.18 \\
\hline \multicolumn{10}{|c|}{ Treatments(T) } \\
\hline $\mathrm{T}_{0}$ & 23.58 & 24.86 & 24.22 & 2.60 & 2.71 & 2.66 & 4.03 & 4.45 & 4.24 \\
\hline $\mathrm{T}_{1}$ & 27.17 & 28.08 & 27.63 & 2.75 & 2.82 & 2.79 & 4.22 & 4.43 & 4.33 \\
\hline $\mathrm{T}_{2}$ & 32.36 & 33.56 & 32.96 & 3.61 & 3.64 & 3.62 & 5.54 & 5.71 & 5.62 \\
\hline $\mathrm{T}_{3}$ & 30.98 & 31.91 & 31.45 & 3.34 & 3.13 & 3.24 & 4.80 & 5.23 & 5.02 \\
\hline $\mathrm{T}_{4}$ & 35.93 & 36.77 & 36.35 & 3.81 & 3.90 & 3.85 & 6.39 & 6.45 & 6.42 \\
\hline $\mathrm{T}_{5}$ & 31.53 & 33.04 & 32.29 & 3.27 & 3.43 & 3.35 & 5.15 & 5.72 & 5.43 \\
\hline $\mathrm{T}_{6}$ & 29.15 & 30.95 & 30.05 & 2.88 & 2.87 & 2.88 & 5.38 & 5.31 & 5.34 \\
\hline $\mathrm{T}_{7}$ & 34.80 & 36.25 & 35.52 & 3.57 & 3.58 & 3.57 & 6.11 & 6.18 & 6.14 \\
\hline $\mathrm{T}_{8}$ & 34.03 & 34.89 & 34.46 & 3.67 & 3.60 & 3.63 & 5.66 & 6.20 & 5.93 \\
\hline $\mathrm{T}_{9}$ & 36.25 & 37.72 & 36.98 & 3.96 & 3.84 & 3.90 & 6.22 & 6.30 & 6.26 \\
\hline S. Em \pm & 0.47 & 0.37 & 0.30 & 0.12 & 0.13 & 0.09 & 0.15 & 0.18 & 0.12 \\
\hline C.D. at $\overline{5 \%}$ & 1.34 & 1.06 & 0.84 & 0.34 & 0.38 & 0.25 & 0.43 & 0.52 & 0.33 \\
\hline \multicolumn{10}{|c|}{ CX T } \\
\hline Candyman $\mathrm{X} \mathrm{T}_{0}$ & 28.43 & 29.50 & 28.96 & 3.27 & 3.10 & 3.19 & 4.18 & 4.25 & 4.21 \\
\hline Candyman $\mathrm{X} \mathrm{T}_{1}$ & 33.59 & 34.37 & 33.98 & 3.41 & 3.25 & 3.33 & 4.39 & 4.25 & 4.32 \\
\hline Candyman $\mathrm{X} \mathrm{T}_{2}$ & 36.75 & 37.57 & 37.16 & 4.02 & 4.36 & 4.19 & 5.48 & 5.31 & 5.39 \\
\hline Candyman $\mathrm{X} \mathrm{T}_{3}$ & 35.46 & 36.64 & 36.05 & 3.92 & 3.87 & 3.89 & 5.24 & 5.11 & 5.17 \\
\hline Candyman $\mathrm{X} \mathrm{T}_{4}$ & 38.52 & 39.79 & 39.16 & 4.09 & 3.96 & 4.03 & 6.83 & 6.37 & 6.60 \\
\hline Candyman $\mathrm{X} \mathrm{T}_{5}$ & 35.93 & 38.12 & 37.02 & 3.78 & 3.47 & 3.62 & 5.56 & 5.48 & 5.52 \\
\hline Candyman $\mathrm{X} \mathrm{T}_{6}$ & 34.65 & 36.08 & 35.37 & 3.51 & 3.45 & 3.48 & 5.80 & 5.22 & 5.51 \\
\hline Candyman $\mathrm{X} \mathrm{T}_{7}$ & 38.37 & 39.47 & 38.92 & 4.17 & 3.92 & 4.05 & 6.64 & 6.27 & 6.45 \\
\hline Candyman $\mathrm{X} \mathrm{T}_{8}$ & 37.49 & 38.61 & 38.05 & 4.21 & 3.93 & 4.07 & 6.00 & 6.50 & 6.25 \\
\hline Candyman $\mathrm{X} \mathrm{T}_{9}$ & 40.63 & 41.41 & 41.02 & 4.17 & 3.93 & 4.05 & 6.77 & 7.16 & 6.97 \\
\hline American Beauty $\mathrm{X} \mathrm{T}_{0}$ & 16.30 & 17.54 & 16.92 & 2.15 & 2.57 & 2.36 & 3.73 & 4.26 & 3.99 \\
\hline American Beauty $\mathrm{X} \mathrm{T}_{1}$ & 17.58 & 18.35 & 17.97 & 2.42 & 2.66 & 2.54 & 3.16 & 3.71 & 3.43 \\
\hline American Beauty X $\mathrm{T}_{2}$ & 20.75 & 22.60 & 21.68 & 3.33 & 3.07 & 3.20 & 4.06 & 4.62 & 4.34 \\
\hline American Beauty X $\mathrm{T}_{3}$ & 19.80 & 20.49 & 20.14 & 2.72 & 2.74 & 2.73 & 3.40 & 3.91 & 3.66 \\
\hline American Beauty $\mathrm{X} \mathrm{T}_{4}$ & 25.91 & 26.37 & 26.14 & 3.32 & 3.48 & 3.40 & 5.32 & 5.56 & 5.44 \\
\hline American Beauty X T ${ }_{5}$ & 20.23 & 21.45 & 20.84 & 2.93 & 3.45 & 3.19 & 3.49 & 4.52 & 4.01 \\
\hline American Beauty $\mathrm{X} \mathrm{T}_{6}$ & 19.13 & 20.34 & 19.74 & 2.32 & 2.50 & 2.41 & 3.68 & 4.14 & 3.91 \\
\hline American Beauty $\mathrm{X} \mathrm{T}_{7}$ & 23.51 & 24.55 & 24.03 & 3.25 & 3.29 & 3.27 & 4.32 & 4.84 & 4.58 \\
\hline American Beauty X T & 22.64 & 23.61 & 23.13 & 3.37 & 3.40 & 3.39 & 4.09 & 4.73 & 4.41 \\
\hline American Beauty X $\mathrm{T}_{9}$ & 24.50 & 26.29 & 25.40 & 3.72 & 3.52 & 3.62 & 4.63 & 4.53 & 4.58 \\
\hline Jester $\mathrm{X} \mathrm{T}_{0}$ & 26.02 & 27.55 & 26.79 & 2.39 & 2.44 & 2.42 & 4.18 & 4.83 & 4.50 \\
\hline Jester $\mathrm{X} \mathrm{T}_{1}$ & 30.35 & 31.51 & 30.93 & 2.41 & 2.56 & 2.48 & 5.12 & 5.33 & 5.23 \\
\hline Jester $\mathrm{X} \mathrm{T}_{2}$ & 39.59 & 40.52 & 40.06 & 3.47 & 3.48 & 3.48 & 7.08 & 7.20 & 7.14 \\
\hline Jester $\mathrm{X} \mathrm{T}_{3}$ & 37.68 & 38.61 & 38.15 & 3.39 & 2.79 & 3.09 & 5.77 & 6.67 & 6.22 \\
\hline Jester $\mathrm{X} \mathrm{T}_{4}$ & 43.35 & 44.14 & 43.74 & 4.02 & 4.25 & 4.13 & 7.01 & 7.41 & 7.21 \\
\hline Jester $\mathrm{X} \mathrm{T}_{5}$ & 38.43 & 39.56 & 39.00 & 3.11 & 3.36 & 3.24 & 6.38 & 7.16 & 6.77 \\
\hline Jester $\mathrm{X} \mathrm{T}_{6}$ & 33.68 & 36.44 & 35.06 & 2.82 & 2.67 & 2.75 & 6.65 & 6.57 & 6.61 \\
\hline Jester $\mathrm{X} \mathrm{T}_{7}$ & 42.51 & 44.72 & 43.62 & 3.29 & 3.52 & 3.41 & 7.37 & 7.42 & 7.40 \\
\hline Jester X T 8 & 41.96 & 42.46 & 42.21 & 3.42 & 3.46 & 3.44 & 6.89 & 7.37 & 7.13 \\
\hline Jester $\mathrm{X} \mathrm{T}_{9}$ & 43.61 & 45.46 & 44.54 & 3.99 & 4.08 & 4.04 & 7.26 & 7.21 & 7.24 \\
\hline S. Em \pm & 0.82 & 0.65 & 0.52 & 0.21 & 0.23 & 0.16 & 0.26 & 0.31 & 0.21 \\
\hline C.D. at $5 \%$ & 2.31 & 1.83 & 1.46 & 0.59 & 0.66 & 0.44 & 0.73 & 0.90 & 0.57 \\
\hline
\end{tabular}


Table.3 Effect of organic resources of nutrients on propagation co-efficient (\%)

\begin{tabular}{|c|c|c|c|}
\hline \multirow{2}{*}{ Treatment Details } & \multicolumn{3}{|c|}{ PROPAGATION CO-EFFICIENT (\%) } \\
\hline & 2009 & 2010 & Pooled \\
\hline Candyman & 223.79 & 235.03 & 229.41 \\
\hline American Beauty & 269.27 & 276.38 & 272.82 \\
\hline Jester & 378.50 & 395.30 & 386.90 \\
\hline S. Em \pm & 1.73 & 5.72 & 2.99 \\
\hline C.D. at $5 \%$ & 4.89 & 16.19 & 8.37 \\
\hline $\mathrm{T}_{0}$ & 159.91 & 165.44 & 162.67 \\
\hline $\mathrm{T}_{1}$ & 229.89 & 233.08 & 231.48 \\
\hline $\mathrm{T}_{2}$ & 322.40 & 337.34 & 329.87 \\
\hline $\mathrm{T}_{3}$ & 254.07 & 259.32 & 256.70 \\
\hline $\mathrm{T}_{4}$ & 353.44 & 360.60 & 357.02 \\
\hline $\mathrm{T}_{5}$ & 282.85 & 321.74 & 302.29 \\
\hline $\mathrm{T}_{6}$ & 215.05 & 217.69 & 216.37 \\
\hline $\mathrm{T}_{7}$ & 304.11 & 310.14 & 307.13 \\
\hline $\mathrm{T}_{8}$ & 350.61 & 354.17 & 352.39 \\
\hline $\mathrm{T}_{9}$ & 432.87 & 462.86 & 447.86 \\
\hline S. Em \pm & 3.15 & 10.44 & 5.45 \\
\hline C.D. at $5 \%$ & 8.92 & 29.57 & 15.27 \\
\hline Candyman $\mathrm{X} \mathrm{T}_{0}$ & 158.19 & 162.38 & 160.28 \\
\hline Candyman $\mathrm{X} \mathrm{T}_{1}$ & 189.66 & 194.40 & 192.03 \\
\hline Candyman $\mathrm{X} \mathrm{T}_{2}$ & 261.73 & 288.31 & 275.02 \\
\hline Candyman $\mathrm{X} \mathrm{T}_{3}$ & 230.73 & 236.65 & 233.69 \\
\hline Candyman $\mathrm{X} \mathrm{T}_{4}$ & 112.15 & 119.66 & 115.91 \\
\hline Candyman $\mathrm{X} \mathrm{T}_{5}$ & 263.96 & 274.05 & 269.00 \\
\hline Candyman $\mathrm{X} \mathrm{T}_{6}$ & 195.75 & 198.24 & 197.00 \\
\hline Candyman $\mathrm{X} \mathrm{T}_{7}$ & 102.70 & 108.73 & 105.72 \\
\hline Candyman $\mathrm{X} \mathrm{T}_{8}$ & 292.48 & 297.16 & 294.82 \\
\hline Candyman $\mathrm{X} \mathrm{T}_{9}$ & 430.56 & 470.76 & 450.66 \\
\hline American Beauty $\mathrm{X} \mathrm{T}_{0}$ & 136.15 & 142.08 & 139.12 \\
\hline American Beauty $\mathrm{X} \mathrm{T}_{1}$ & 174.14 & 177.33 & 175.73 \\
\hline American Beauty $\mathrm{X} \mathrm{T}_{2}$ & 280.90 & 287.82 & 284.36 \\
\hline American Beauty $\mathrm{X} \mathrm{T}_{3}$ & 215.88 & 220.68 & 218.28 \\
\hline American Beauty $\mathrm{X} \mathrm{T}_{4}$ & 434.35 & 441.13 & 437.74 \\
\hline American Beauty $\mathrm{X} \mathrm{T}_{5}$ & 227.07 & 230.06 & 228.57 \\
\hline American Beauty $\mathrm{X} \mathrm{T}_{6}$ & 184.52 & 188.69 & 186.60 \\
\hline American Beauty $\mathrm{X} \mathrm{T}_{7}$ & 337.94 & 343.51 & 340.73 \\
\hline American Beauty $\mathrm{X} \mathrm{T}_{8}$ & 318.29 & 321.25 & 319.77 \\
\hline American Beauty $\mathrm{X} \mathrm{T}_{9}$ & 383.46 & 411.20 & 397.33 \\
\hline Jester $\mathrm{X} \mathrm{T}_{0}$ & 185.39 & 191.85 & 188.62 \\
\hline Jester X T 1 & 325.86 & 327.51 & 326.69 \\
\hline Jester $\mathrm{X} \mathrm{T}_{2}$ & 424.56 & 435.89 & 430.22 \\
\hline Jester $\mathrm{X} \mathrm{T}_{3}$ & 315.61 & 320.62 & 318.12 \\
\hline Jester $\mathrm{X} \mathrm{T}_{4}$ & 513.81 & 520.99 & 517.40 \\
\hline Jester $\mathrm{X} \mathrm{T}_{5}$ & 357.52 & 461.10 & 409.31 \\
\hline Jester $\mathrm{X} \mathrm{T}_{6}$ & 264.89 & 266.14 & 265.52 \\
\hline Jester $\mathrm{X} \mathrm{T}_{7}$ & 471.70 & 478.19 & 474.95 \\
\hline Jester $\mathrm{X} \mathrm{T}_{8}$ & 441.06 & 444.10 & 442.58 \\
\hline Jester $\mathrm{X} \mathrm{T}_{9}$ & 484.57 & 506.62 & 495.60 \\
\hline S. Em+ & 5.46 & 18.08 & 9.44 \\
\hline C.D. at $5 \%$ & 15.45 & 51.21 & 26.46 \\
\hline
\end{tabular}


In the present investigation, American Beauty with $\mathrm{T}_{4}$ treatment $(1 / 2 \mathrm{FYM}+1 / 2$ vermicompost + bone meal $5 \mathrm{t} / \mathrm{ha}+$ Trichoderma $20 \mathrm{~g} / \mathrm{m}^{2}$ as basal) was found to be better in number of corms per plant. Rama et al., (2005) conducted an experiment to evaluate the performance of 8 cultivars. As per their investigation, White Prosperity was recorded the highest number of corms (1.79) per plant. Barman et al., (2006) also studied the effect of bio-fertilizer on corm production in gladiolus. He founded that FYM and leaf mould was found to have the highest result of corm production compared to untreated controls. Godse et al., (2006) also reported maximum number of corms per plant was found when plants were treated with vermicompost $8 \mathrm{t} / \mathrm{ha}+$ Azotobacter amd PSB @ $25 \mathrm{~kg} / \mathrm{ha}$ each $+80 \%$ recommended dose of fertilizer. Candyman with $\mathrm{T}_{9}$ combination of treatment was found to have the highest cormels yield per plant. $\mathrm{T}_{9}$ treatment was found to be the most effective when combined with varieties. Gangadharan and Gopinath (2000) found that combination of vermicompost $+80 \%$ NPK resulted in the maximum number of cormels per plant.

Kathiresan and Venkatesh (2002) also reported in gladiolus that combined application of Azospirillum and VAM along with recommended dose of NPK increase the number of cormels per plant. Maximum weight of corm was recorded with Jester and $\mathrm{T}_{4}$ treatment $(1 / 2 \mathrm{FYM}+1 / 2$ vermicompost + bone meal $5 \mathrm{t} / \mathrm{ha}+$ Trichoderma $20 \mathrm{~g} / \mathrm{m}^{2}$ as basal). Jester and $\mathrm{T}_{9}$ treatment was found to have the highest weight of cormels per plant as compared to other varieties with different treatments. Gangadharan and Gopinath (2000) similarly reported that application of vermicompost at the rate of 10 tonnes per hectare along with $80 \%$ RDF of NPK significantly increased weight of corm and cormels per plant in gladiolus variety White Prosperity. The highest polar diameter of corms was found with Candyman and $\mathrm{T}_{2}$ treatment (FYM 50t/ha + bone meal 5t/ha + Trichoderma $20 \mathrm{~g} / \mathrm{m}^{2}$ as basal). Altaff et al., (2002) also reported that the highest onion bulb diameter was found when it was treated with Azospirillum and Azotobacter. Fentahun and Singh (1999) recorded the highest bulb horizontal diameter in onion when they were treated with Azospirillum + VAM $+25 \mathrm{~kg}$ phosphorus in their experiment. Gangadharan and Gopinath (2000) also reported that diameter of corm was the highest when treated with combination of vermicompost at 10 tonnes/ha $+80 \%$ recommended NPK rate as compared to other combination. The highest equatorial diameter of corm was found with Jester and $\mathrm{T}_{7}$ treatment (Phosphobacteria $2 \mathrm{~g} /$ plant + bone meal 5t/ha + Trichoderma 20g/m2 as basal dose). Azospirillum is a free living diazotroph, apart from nitrozen fixing ability and it is known to produce auxins, cytokinins and gibberellins (Tien et al., 1979). Phosphate solubilizers containing mainly Pseudomonas, Bacillus and Aspergillus etc. have the abilities to solubilize insoluble phosphorus to plants (Gaur, 1985). Subbiah (1991) also suggested that biofertilizers are micro-organisms that are capable of mobilizing nutritive elements form non available form to useable from through biological processes.

So, application of bone meal along with phospho-bacteria was found to be effective in releasing phosphorus from bone meal slowly to available form. Gangadharan and Gopinath (2000) also found significant increase in equatorial diameter of corm because of application of vermicompost at the rate 10 tonnes/ha along with $80 \%$ recommended dose of NPK fertilizer. Similarly Teran et al., (1994) reported that Azotobacter + fillar cake @180 tonnes/ha and Glomus Faciculatum increased the bulb size in onion. Fentahun and Singh (1999) recorded the highest bulb horizontal and vertical diameter with the 
application of Azospirillum + VAM + 50N . The salient features of the present investigation are summarized as follows:

Candyman was found highest number of cormels per plant, highest propagation Coefficient, when treated with VAM, Azospirillum, phosphobacteria, bone meal and Trichoderma.

American Beauty was found to have maximum number of corms per plant when treated with $1 / 2 \mathrm{FYM}+1 / 2$ vermicompost, bone meal $(5 \mathrm{t} / \mathrm{ha})+$ Trichoderma $\left(20 \mathrm{~g} / \mathrm{m}^{2}\right)$ and Jester produced the highest weight of the corm with the same treatment.

Polar diameter of corm was found highest in the variety Candyman with FYM (50t/ha), bone meal $(5 \mathrm{t} / \mathrm{ha})$ and Trichoderma $\left(20 \mathrm{~g} / \mathrm{m}^{2}\right)$ as basal among all other combinations. Equatorial diameter was found highest in Jester with the treatment of phospho-bacteria (2g/plant), bone meal (5t/ha) and Trichoderma $\left(20 \mathrm{~g} / \mathrm{m}^{2}\right)$ as basal among all other combination of treatments.

Among the ten treatments related to provide nutrition to gladiolus cultivars application of VAM (2g/plant) + Azospirillum (2g/plant) + Phosphobacteria (2g/plant) + bone meal $(5 \mathrm{t} / \mathrm{ha})+$ Trichoderma $\left(20 \mathrm{~g} / \mathrm{m}^{2}\right)$ when applied as basal showed better performance in respect of several morphological and quality parameters so the treatment might be chosen and recommended for all round performance of the gladiolus variety Jester and Candyman under hilly region of West Bengal.

\section{References}

Altaff, H.A., O.S. Saeed and A. Z. Salim, 2002. Effect biofertilizer, inorganic and foliar application of power 4 on the productivity of onion. University of Aden. J. Natural Applied Science, 6(1):
$1-4$

Barman, D., Rajni, K., Pal, R. and Upadhyaya, R.C. 2006. Studies on corm and cormel production in gladiolus. Journal of Ornamental Horticulture. 9(2): 118-121

Fentahun Mengistu and Narendra Singh, 1999. Effect of biofertilizers on growth, yield and nutrient uptake of onion (Allium cepa). Veg. Sci., 26 (2): 193195

Gangadharan, G.D. and Gopinath, G. 2000. Effects of organic and inorganic fertilizers on yield of spikes, corms, cormels and returns of gladiolus cv. White Prosperity. Karnataka-Journalof-Agricultural-Sciences. 13(4): 937941

Gaur, A.C. 1985. Phosphate solubilizing micro-organisms and their role in plant growth and crop yield. Proc. Natl. Symp. On Soil Biol., Hissar, pp. 125138

Godse, S.B.; Golliwar, V.J.; Neha Chopde; Bramhankar, K.S. and Kore, M.S. 2006. Effect of organic manures and biofertilizers with reduced doses of inorganic fertilizers on growth, yield and quality of gladiolus. Journal-ofSoils-and-Crops. 16(2): 445-449

Gomez, K.A. and Gomez, A.A. 1984. Statistical procedures for Agricultural Research. John Wiley and Sons, New York

Kathiresan, C. And J. Venkatesh, 2002. Effect of biofertilizer with levels of $\mathrm{N}$ and $\mathrm{P}$ on gladiolus. In: Floriculture Research Trends in India, pp. 118-121

Rama, R. B.; Tomar, K. S. and Datta, S. K.2005. Performance of certain gladiolus varieties under sodic conditions. Journal-of-OrnamentalHorticulture-New-Series. 8(1): 77-78

Subbiah, K. 1991. Studies on the effect of nitrogen and Azospirillum on okra. South Indian Hort., 39 (1): 37-41 
Teran, Z., R. Espiosa, F. Fermandez and G. Gras, 1994. Application of biofertilizers and filter press cake in the production of seedlings of the onion (Allium cepa L.). cv. Red Creole. Cultivos-Tropicales. 15(1): $32-35$
Tien, T.M.; Gaskins, M.H. and Hubbell, D.H. 1979. Plant growth substances produced by Azospirillum brasilense and their effect on the growth of pearl millet (Pennisetum americamum L.). Applied Environmental Micro-biology. 37: 1016-1024

\section{How to cite this article:}

Sarkar, I., S. Maitra and Swathi, K. 2017. Effect of Organic Resources of Nutrients for Corms and Cormlet Production of Three Cultivars of Gladiolus (Gladiolus grandiflorus L.) in the Hilly Tract of West Bengal. Int.J.Curr.Microbiol.App.Sci. 6(8): 1572-1582. doi: https://doi.org/10.20546/ijcmas.2017.608.188 